\title{
Light-Hierarchies: Optimal Multicast Routes under Optical Constraints
}

\author{
Bernard Cousin $^{\mathrm{a}}$, Miklos Molnar ${ }^{\mathrm{b}}$, Fen Zhou ${ }^{\mathrm{c}}$ \\ ${ }^{a}$ University of Rennes I - IRISA, Campus de Beaulieu, 35042 Rennes Cedex, France \\ ${ }^{\mathrm{b}}$ University of Montpellier 2 - LIRMM, 161 rue Ada, 34095 Montpellier Cedex 5, France \\ ${ }^{\mathrm{c}}$ Telecom Bretagne, CS 83818, 29238 BREST Cedex 3, France
}

\begin{abstract}
Multicast routing in all optical WDM networks where the light splitting capacity of some optical switches is limited is an important problem. The computation problem of the minimum cost multicast routes under optical constraints is NP-difficult. To solve the optimal multicast routing problem under physical constraints, we propose a new routing structure called 'light-hierarchy". In a light-hierarchy, the multicast route can traverse the same optical switch several times using the same wavelength. This new routing structure may improve both the cost of the optical structures and the throughput on WDM multicast networks.
\end{abstract}

\section{INTRODUCTION}

Multicast routing in all optical WDM networks where the light splitting capacity of some optical switches is limited (due to the cost and additional complexity of optical switch with light splitters) is an important problem $[1,9,13]$. To compute multicast routes, most of the multicast algorithms propose to use "light-trees" or a set of trees called "lightforest" [3, 4, 7, 11, 12]. More recently, "light-trails" were also proposed [14] to decrease the network resource consumption using non elementary light-paths. However, these later optical routes do not easily fit in a multicast tree or to a set of trees. The computation problem of the minimum cost multicast routes under optical constraints (which is NP-difficult) needs a deep analysis. To solve the optimal multicast routing problem under physical constraints, a new structure is proposed, which is called "hierarchy". For optical routing, a "light-hierarchy" [1, 13] corresponds to a hierarchy of successive neighbor nodes which use one wavelength. Surprisingly in a light-hierarchy, the multicast route can traverse the same optical switch several times using the same wavelength. This new routing proposition may improve both the cost of the optical structures and the throughput on WDM multicast networks. Besides the identification of the minimum cost optical multicast routes, our analysis will permit to propose fast heuristics computing low cost light-hierarchies.

\section{LIGHT-HIERARCHY}

To support multicast in all-optical networks, network nodes should be equipped with optical power splitters, which is capable of splitting the incoming light signal into all the outgoing ports. Thus they are called multicast capable nodes (MC) [4]. However, when a light signal is split into $\mathrm{m}$ copies, the signal power on each outgoing port will be reduced to or less than $1 / \mathrm{m}$ of the incoming signal power [5]. Power loss, complicated architecture plus expensive fabrication prevent the availability of splitters on all network nodes. A multicast incapable (MI) node is incapable of light splitting (but they may benefit of the Tap and Continue capacity). In absence of wavelength converter, the same wavelength should be retained along all the light-path. Therefore, the MI nodes were thought to be able to only act either as a leaf node or as a two degree intermediate node in a light-tree (if the Tap and Continue capability is available at the MI nodes). Nevertheless, it is very interesting to find that an MI node can work as a special branching node by using Cross Pair Switching (CPS).

For instance, let two signals on the same wavelength wo come from two different light-paths, and enter two different input ports of an MI node. Due to their internal switching architecture, some of the MI nodes may be able to switch these two signals into two outgoing ports without any conflict. Note that the signals are still on the same wavelength wo, and should mandatory be forwarded to different successor nodes.

Based on the CPS capacity of MI nodes, an MI node could connect two or more successor nodes in a light structure by making use of different input and output port pairs. In this case, an MI node can be traversed several times, thus the multicast structure will be no longer a lighttree, but a light-hierarchy, where cycles may exist.

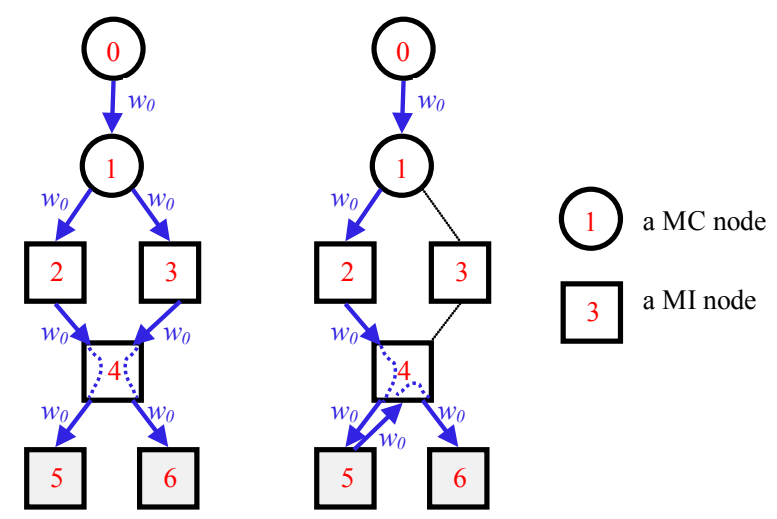

Fig. 1. Two typical light-hierarchies with Cross Pair Switching 
Two typical light-hierarchies with Cross Pair Switching are demonstrated in Fig. 1, where the circle denotes an MC node while the rectangle stands for an MI node. Source node 0 multicast messages to destination nodes 5 and 6 . In the left hierarchy of Fig. 1, the light signal emitted by node 0 is split into 2 copies by MC node 1, then these two copies enter two different incoming ports of MI node 4 and are switched to destination nodes 5 and 6 respectively. This kind of Cross Pair Switching benefits from the high degree of MI node 4 (the degree node must at least of 4). In the right hierarchy of Fig. 1 the light signal first goes out from MI node 4 to destination node 5 and returns back to node 4 . The light signal is then forwarded to destination node 6. This Cross Pair Switching is based on the simultaneous usage of two oppositely directional fiber links. Cross Pair Switching is neither always available nor useful, but anyhow a light-tree structure can be viewed as a light-hierarchy without cycle.

Thus a light-hierarchy is a set of consecutive and directed fiber links occupying the same wavelength, which is rooted from the source and terminated at some of the destinations. Light-hierarchies can offer flexible solutions for optical multicast routing taking into account typical optical constraints (continuity of the wavelength in light-paths, scarcity of splitters in network). For instance, an MI node cannot be a branching node but, in a light-hierarchy, it can be traversed twice using the same wavelength. So, some MI nodes, which are not solicited in a light-tree (because they are authorized to have at most one outgoing path), can be used in a light-hierarchy to reach several destinations which are on different outgoing paths. Differently from a light-tree, light-hierarchy is free of the non- repetition of nodes while it still forbids the duplicate use of the same directed link. It can be expressed as an enumeration of nodes and links, for instance the light-hierarchy of left part of Fig. 1 can be specified by $\mathrm{LH}=0\left(\mathrm{I}_{01}, 1\left(\mathrm{I}_{12}, 2\left(\mathrm{I}_{24}, 4\left(\mathrm{I}_{45}, 5\right)\right), \mathrm{I}_{13}, 3\left(\mathrm{I}_{34}\right.\right.\right.$, $\left.\left.\left.4\left(\mathrm{I}_{46}, 6\right)\right)\right)\right)$. In this example, node 4 is listed twice.

A light-hierarchy has the following properties:

(a) Each link is directed and can be used only once. Thus in a light-hierarchy, only one wavelength is used over any network links.

(b) Between each pair of nodes in a light-hierarchy, there are at most two links in condition that they are used for opposite direction communications.

(c) Each link has one and only one predecessor link, except that the links coming from source.

(d) Nodes can be used several times. So, cycles are permitted if we project the light-hierarchy onto the network graph.

(e) Each occurrence of a node in a light-hierarchy respects the maximum number of output links due to the splitting capacity of the node. The number of input and output links of a node varies according to its splitting capacity.

For a non-terminal MI node, multiple incoming links are allowed. However, each incoming link should correspond to a distinct outgoing link. Hence, the number of input links of a non-terminal MI node should be equal to that of its output links. Besides, an MC node (with remaining splitting capacity) should have one and only one input link while it can have as many output links as it can. Since a node can be present several times in the light-hierarchy, a destination node can also be repeated. In such a case, the destination node must receive the messages for local usage only once.

\section{PERformance EVAluation}

To show the advantage of our light-hierarchy (LH) structure, simulation is conducted to compare it with the light-tree (LT) structures. We have used two well known network topologies: the NSF network with 14 nodes and the European Cost-239 network with 11 nodes. Given a group size $|\mathrm{D}|, 100$ random multicast sessions have been generated. The membership of each multicast session follows a uniform distribution in the topology. Then, we use integer linear programming (ILP) method to search the optimal light-trees and the light-hierarchies with the minimum cost for each multicast session. A complete ILP formulation of the problem can be found in [13].

We take into account the following metrics:

(a) Overall cost (i.e. the sum of the light channels over all the links used), for the establishment of all the sessions. We give also the cost saving percentage of light-hierarchy structure compared to light-tree structure.

(b) The number of wavelengths required, for all the sessions.

(c) $\mathrm{CPS} \%$, the percentage of light-hierarchies in which the Cross Pair Switching capability is employed by at least one node, for all the sessions.

The numerical results are presented for NSF network (in Table I) and for COST-238 network (in Table II). Two cases are considered for the NSF network: without splitter and with sparse splitting (two nodes are splitters). Based on the simulation results, it is observed that:

(1) The proposed light-hierarchy structure always achieves much lower total cost than the traditional light-tree structure. The cost can be saved up to $3.6 \%$ by 57 lighthierarchies with CPS in NSF network, while up to $6.2 \%$ by 81 light-hierarchies with CPS in Cost-239 network. Therefore, light-tree structure is not optimal from the point view of cost, but the light-hierarchy structure can be better.

(2) In general, the absolute cost reduction by the lighthierarchy structure depends on the number of Cross Pair Switching used, i.e. R(CPS). This is because that with the help of Cross Pair Switching of MI nodes a destination may connect to the light-hierarchy with less cost by using cycles.

(3) Fewer wavelengths on average are required for establishing 100 multicast sessions, when the light-hierarchy method is adopted.

All of these advantages benefit from the proposed Cross Pair Switching capability of nodes. The light-tree structure requires that each node should have only one input link, while the light-hierarchy structure accepts cycles (several input links). Since the new light-hierarchy structure overcomes the inherent shortcoming of the tree structure, more destination nodes can be served in one light-hierarchy than a light-tree, and thus fewer wavelengths is required by 
each session. With the help of the light-hierarchy structure, a destination node is more likely to connect to the nearest node (even if it is an MI node) in the light-hierarchy while it may have to lead a long way to the source node on another wavelength in order not to violate the light-tree structure. As the light-tree is a special type of light-hierarchy, the optimal light-hierarchy solution at least has the same cost as the light-tree solution in the worst cases. Once useful Cross Pair Switching node is found, the total cost is decreased. More Cross Pair Switching is used, more cost will be saved. This explains the third observation.

TABLE I: PERFORMANCE EVALUATION IN USA NSF NETWORK.

\begin{tabular}{|c|c|c|c|c|c|c|}
\hline \multicolumn{7}{|c|}{ No splitter } \\
\hline Size & \multicolumn{3}{|c|}{ Overall Cost } & \multicolumn{2}{|c|}{ \# Wavelengths } & $\mathrm{LH}$ \\
\hline$|\mathrm{D}|$ & $\mathrm{LH}$ & LT & $\begin{array}{l}\text { (LT-LH) } \\
\text { /LT (\%) }\end{array}$ & $\mathrm{LH}$ & LT & CPS $\%$ \\
\hline 2 & 2059 & 2079 & 0.96 & 103 & 106 & 10 \\
\hline 6 & 4096 & 4247 & 3.56 & 107 & 114 & 35 \\
\hline 9 & 5025 & 5213 & 3.61 & 115 & 147 & 57 \\
\hline 13 & 6237 & 6330 & 1.47 & 121 & 156 & 67 \\
\hline \multicolumn{7}{|c|}{ Two nodes are splitters } \\
\hline Size & \multicolumn{3}{|c|}{ Overall Cost } & \multicolumn{2}{|c|}{ \# Wavelengths } & $\mathrm{LH}$ \\
\hline$|\mathrm{D}|$ & $\mathrm{LH}$ & LT & $\begin{array}{l}\text { (LT-LH) } \\
/ \mathrm{LT}(\%)\end{array}$ & $\mathrm{LH}$ & $\mathrm{LT}$ & CPS $\%$ \\
\hline 2 & 2055 & 2075 & 0.96 & 103 & 106 & 11 \\
\hline 6 & 4017 & 4080 & 1.54 & 105 & 108 & 32 \\
\hline 9 & 4898 & 4984 & 1.73 & 105 & 112 & 36 \\
\hline 13 & 6035 & 6035 & 0 & 106 & 111 & 5 \\
\hline
\end{tabular}

TABLE II: PERFORMANCE EVALUATON IN EUROPEAN COST-239 NETWORK.

\begin{tabular}{|c|c|c|c|c|c|c|}
\hline \multicolumn{7}{|c|}{ Two node are splitters } \\
\hline Size & \multicolumn{3}{|c|}{ Overall Cost } & \multicolumn{2}{|c|}{ \# Wavelengths } & $\mathrm{LH}$ \\
\hline$|\mathrm{D}|$ & LH & LT & $\begin{array}{l}\text { (LT-LH) } \\
/ \text { LT }(\%)\end{array}$ & LH & LT & CPS $\%$ \\
\hline 2 & 1329 & 1344 & 1.12 & 100 & 108 & 16 \\
\hline 5 & 2685 & 2863 & 6.22 & 102 & 183 & 82 \\
\hline 7 & 3580 & 3747 & 4.46 & 100 & 223 & 93 \\
\hline 10 & 5204 & 5280 & 1.44 & 100 & 272 & 100 \\
\hline
\end{tabular}

\section{CONCLUSION}

We propose in this paper a new all-optical multicast structure, called light-hierarchy. It improves the quality of multicast routing in sparse splitting WDM networks. A lighthierarchy is a set of consecutive and directed fiber links occupying the same wavelength, which is rooted from the source and terminated at some destinations. A same node may appear several times in a light-hierarchy. Different from a light-tree, a light-hierarchy structure accepts cycles. It benefits of the Cross Pair Switching capability of MI nodes: an MI node may to serve several destination nodes on the same wavelength through its different input and output pairs. Light-hierarchy structure overcomes the inherent drawback of the traditional light-tree structure, so that the splitting constraint is relaxed to some extent. This is why it outperforms the light-tree in term of cost. We showed that the optimal multicast structure for minimizing the wavelength channel cost is not a set of light-trees, but a set of light-hierarchies rooted at the source. Numerical results verified that the light-hierarchy structure is the cost optimal solution for all-optical multicast routing with sparse splitting constraint. Using light-hierarchies, fewer wavelengths on average are required for establishing a set of multicast sessions.

\section{REFERENCES}

[1] Fen Zhou, Miklos Molnar, Bernard Cousin, "Light-Hierarchy: The Optimal Structure for Multicast Routing in WDM Mesh Networks," In Proc. IEEE Symposium on Computers and Communications. Riccione, Italia. 22-25 June, 2010.

[2] Maria Ramalho, "Intra- and Inter-domain Multicast Routing Protocols: a Survey and Taxonomy," IEEE Communications Surveys \& Tutorials, 3(1): 2-25, 2000.

[3] Jingyi He, S.-H. Gary Chan, and Danny H.K. Tsang, "Multicasting in WDM Networks," IEEE Communication Surveys \& Tutorials, 4(1): 2-20, 2002.

[4] Rajkumar Malli, Xijun Zhang, Chunming Qiao, "Benefit of Multicasting in All-optical Networks," SPIE Proceeding on AllOptical Networking, 2531: 209-220, 1998.

[5] Maher Ali, Jitender S. Deogun, "Power-efficient Design of Multicast Wavelength Routed Networks," IEEE Journal on Selected Areas in Communications, 18(10): 1852-1862, 2000.

[6] Maher Ali, Jitender S. Deogun, "Cost-effective Implementation of Multicasting in Wavelength-Routed Networks," IEEE/OSA Journal of Lightwave Technology, 18(12): 1628-1638, 2000.

[7] Laxman H. Sahasrabuddhe, Biswanath Mukherjee, "Light-trees: Optical Multicasting for Improved Performance in WavelengthRouted Networks," IEEE Communications Magazine, 37(2): 67-73, 1999

[8] Xiaohua Jia, Dingzhu Du, Xiaodong Hu, Mankei Lee, Jun Gu, "Optimization of Wavelength Assignment for QoS Multicast in WDM Networks." IEEE Transaction on Communications, 49(2): 341-350, 2001.

[9] Xijun Zhang, John Y. Wei, Chunming Qiao, "Constrained Multicast Routing in WDM Networks with Sparse Light Splitting." IEEE/OSA Journal of Lightwave Technology, 18(12): 1917-1927, 2000.

[10] Ashraf M. Hamad, Ahmed E. Kamal, "Optimal Power-aware Design of All-optical Multicasting in Wavelength Routed Networks," In Proc. IEEE International Conference on Communications, pp. 1796 - 1800, Vol.3, 2004.

[11] L. H. Sahasrabuddhe, and B. Mukherjee, "Light-Trees: Optical Multicasting for Improved Performance in Wavelength-Routed Networks," IEEE Communications Magazine, vol. 37, no. 2, pp. 6773, Feb. 1999.

[12] Ming-Tsung Chen, B.M.T. Lin, Shian-Shyong Tseng, "Multicast Routing and Wavelength Assignment with Delay Constraints in WDM Networks with Heterogeneous Capabilities," Journal of Network and Computer Applications, 31:47-65, 2008.

[13] Fen Zhou, Miklos Molnar, Bernard Cousin, "Is Light-tree Structure Optimal for Multicast Routing in Sparse Light Splitting WDM Networks," In Proc. $18^{\text {th }}$ International Conference on Computer Communications and Networks, San Francisco, USA, 2009.

[14] Yan Li, Jianping Wang, Ashwin Gumaste, Yun Xu, Yinlong Xu, "Multicast Routing in Light-trail WDM networks," In proceedings of IEEE Globecom, p1-5, 2008.

[15] Yufeng Xin and George N. Rouskas, "Multicast Routing under Optical Layer Constraints," in Proc. IEEE INFOCOM, 2004, vol. 4, pp. 2731-2742, 2004.

[16] De-Nian Yang, and Wanjiun Liao, "Design of Light-Tree Based Logical Topologies for Multicast Streams in Wavelength Routed Optical Networks," in Proc. IEEE INFOCOM, vol. 1, pp. 32-41, 2003. 